\title{
Dislocation Content of Non-Classical Twin Boundaries in Hexagonal Metals
}

\author{
V. PAIDAR ${ }^{a, *}$, A. Ostapovets ${ }^{b}$ AND T. KÁŇA ${ }^{b}$ \\ ${ }^{a}$ Institute of Physics, Academy of Sciences of the Czech Republic, \\ Na Slovance 2, CZ-182 21 Prague 8, Czech Republic \\ ${ }^{b}$ Central European Institute of Technology-Institute of Physics of Materials (CEITEC IPM), \\ Academy of Sciences of the Czech Republic, Žižkova 22, CZ-616 62 Brno, Czech Republic
}

\begin{abstract}
Dislocation content of various interfaces in twinned hexagonal crystals has been studied. The analysis of their crystallography shows that the so-called disconnections play an essential role and determine the properties of special planar defects in hexagonal materials.
\end{abstract}

DOI: 10.12693/APhysPolA.134.692

PACS/topics: 61.72.Bb, 61.72.Mm, 62.20.F-

\section{Introduction}

Deformation twins are frequently observed in metals with hexagonal close packed structure and their alloys [1], in particular, for the loading along the $\langle c\rangle$ axis, when the dislocation glide along the basal and prismatic planes does not take place. The room for twinning activity occurs due to relative hardness of non-basal slip, for example, in magnesium [2, 3]. Several twinning modes are observed in magnesium, however, the most frequent one is so-called "tension" twinning on the twinning plane $(10 \overline{1} 2)$ (K1) with the twinning direction [1011] ( $\eta 1)$ activated for tensile straining. There is, however, another symmetrically related so-called conjugate twinning plane $(10 \overline{1} \overline{2})$. Both these twins are misoriented with respect to the matrix crystal about $86^{\circ}$ about the common rotation axis $[1 \overline{2} 10]$ in the plus and minus senses. Generally, for the loading along the $\langle c\rangle$ axis, six different twins of the $\{10 \overline{1} 2\}$ type can be activated with the same probability. Nevertheless, we will consider here below only the

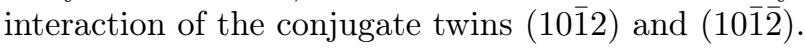

The $c$ and $a$ are hexagonal lattice parameters. The ideal $c / a$ ratio $(4 / \sqrt{ } 6=1.633)$ can be defined as that where the distances between all nearest neighbours in a hexagonal lattice are of the same magnitude. The value for magnesium (1.624) is close to it.

Despite a great effort to understand deformation twinning, some details of twin interactions are still lacking. For instance, interaction of two growing twins can lead to occurrence of new interfaces in materials. These interfaces are different from classical twin boundaries. The conditions of their formations and their properties are still a subject of intensive research. Recently, some investigations on twin-twin boundaries in magnesium were presented by $\mathrm{Yu}$ et al. $[4,5]$. It was shown that the twin-

*corresponding author; e-mail: paidar@fzu.cz twin boundaries formed by the interaction between two $\{10 \overline{1} 2\}$ twin variants sharing the same [1 $\overline{2} 10]$ zone axis were nearly parallel to the (0002) basal plane or (10 $\overline{1} 0)$ prismatic plane of the twin crystals. Such interfaces were also observed experimentally in [6]. Another type of twintwin boundaries was observed in [7], where interaction of two $\{10 \overline{1} 2\}$ twin variants leads to nucleation of $(11 \overline{2} 2)$ or $(11 \overline{2} 6)$ boundaries. Our attention is concentrated in this short contribution only on certain special interfaces that can appear during twinning processes.

\section{Basal/prismatic interfaces}

Basal/prismatic interfaces are often observed in connection to the $\{10 \overline{1} 2\}$ twin boundaries [8-10]. It has been shown in [11] that the symmetric conjugate twin boundaries TwB, limiting a twin embryo (10 $\overline{1} 2)$, are connected by the asymmetric interfaces between the matrix and twin on the basal and prismatic planes BPI and PBI (see Fig. 1). The first letter $\mathrm{B}$ or $\mathrm{P}$ indicates which atomic plane is outside the embryo in the matrix. The symmetric twin boundaries are not exactly mutually perpendicular, there is a small misorientation between them of only about $4^{\circ}$. Notice that two types of such interfaces basal/prismatic or prismatic/basal are equivalent. It has been demonstrated that the energy of these interfaces is of the same order of magnitude as the energy of symmetric twin boundaries. Hence, in the projection along the $\langle a\rangle$ lattice vector, the shape of the twin is roughly an eight-sided polyhedron bordered by four symmetric and four asymmetric interfaces.

The misfit between the basal and prismatic planes, given by the atom separations $c$ and $a \sqrt{ } 3$, is about $6 \%$ (5.7\% for ideal $c / a$ ratio and $6.2 \%$ for $\mathrm{Mg}$ ). There is no difference in atomic spacing along the [12 10$]$ direction for the interfaces with the common [12 10$]$ direction considered here. According to [12], the Burgers vectors of twinning dislocations, $\boldsymbol{b}_{\boldsymbol{t}}$, can be determined from the dichromatic complex to have a magnitude of about $0.14 a$ in the $[\overline{1} 011]$ direction. A series of these dislocations on 


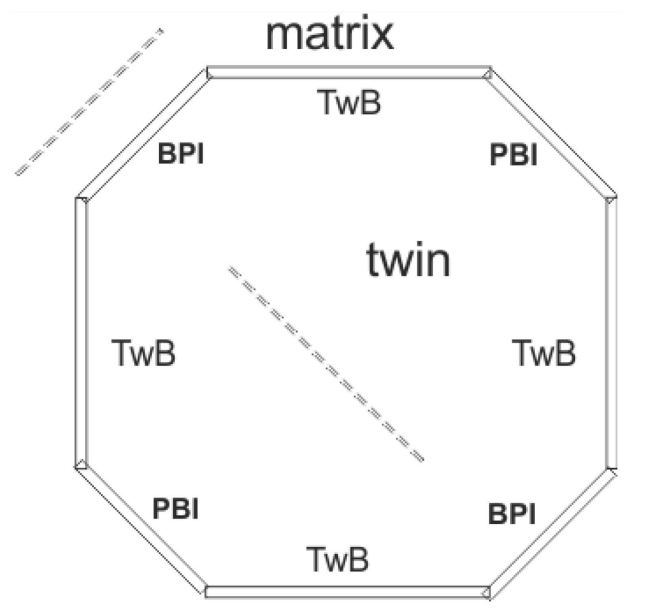

Fig. 1. The shape of the (1012) twin embryo. Two pairs of the conjugate $(10 \overline{1} 2)$ twin boundaries are denoted as TwB and four segments of the basal/prismatic interfaces as BPI or PBI. The projections of the basal planes in the twin and surrounding matrix are marked by dashed lines.

the regular steps of disconnections along the interface of the height about $1.19 a$ can compensate the misfit of $6 \%$ on the planes inclined roughly about $45^{\circ}$ to the symmetric twin boundary TwB (see Fig. 2). This is a plane of the BP interface. Notice that the twin in the $[1 \overline{2} 10]$ projection can be limited by conjugate symmetrical TwB inclined roughly only $90^{\circ}$ as shown in Fig. 1 . The Burgers vectors of edge twinning dislocations $\boldsymbol{b}_{\boldsymbol{t}}$ can be decomposed into two shorter Burgers vectors $\boldsymbol{b}_{\boldsymbol{b}}$ and $\boldsymbol{b}_{\boldsymbol{p}}$, where $\boldsymbol{b}_{\boldsymbol{b}}$ are adding the basal atomic planes to the lattice and $\boldsymbol{b}_{\boldsymbol{p}}$ are adding the prismatic atomic planes. The series of $\boldsymbol{b}_{\boldsymbol{p}}$ dislocations at the distances of about $1.68 a$ form a small deviation from the prismatic planes in the twin of about $3.4^{\circ}$.

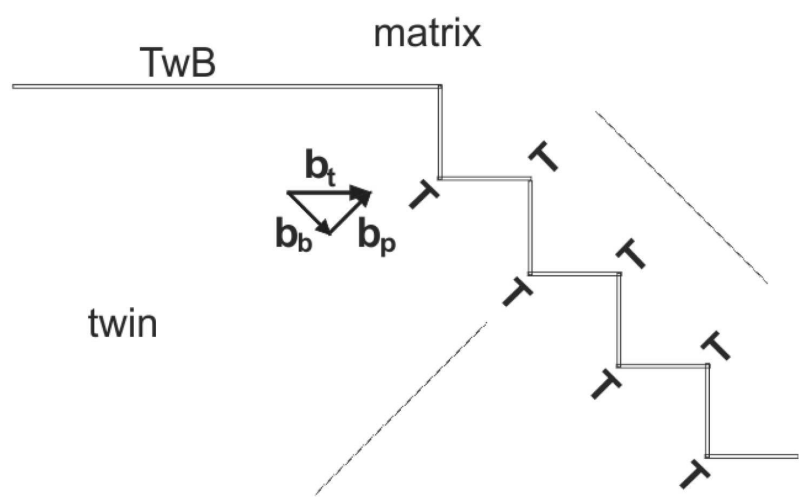

Fig. 2. Junction of the symmetric twin boundary TwB and the basal/prismatic asymmetric interface that is formed by steps of disconnections. Burgers vectors of twinning dislocations and its components are denoted as $\boldsymbol{b}_{\boldsymbol{t}}$ and $\boldsymbol{b}_{\boldsymbol{b}}, \boldsymbol{b}_{\boldsymbol{p}}$. The projections of the basal planes in the twin and surrounding matrix are marked by dashed lines.

\section{Conjugate twin junctions}

When the conjugate twins Tw1 and Tw2 encounter one with the other on the boundary plane with approximately prismatic plane orientation in both twins, the misorientation is about $7^{\circ}\left(6.7^{\circ}\right.$ for the ideal $c / a$ ratio and $7.4^{\circ}$ for $\mathrm{Mg}$ ) and so such interface is a low angle grain boundary. The basal planes in these two twins are misoriented with respect to the matrix by about plus and minus $86^{\circ}$. The orientation of basal planes is marked in all three crystals in Fig. 3 and it is obvious that the interfaces between both twins and the matrix are of the BP type.

The crystallographic relations between the matrix and two conjugate twins are depicted in Fig. 3. The interface between the twins is denoted as prismatic/prismatic as it is close to the prismatic planes orientations in both twins. Both interfaces between the twins and the matrix are of similar character and their orientations are close to the basal planes in the twins and close to the prismatic plane in the matrix. A symmetrical TwB would appear between the matrix and the twin only if the interface plane here would be inclined about $45^{\circ}$ or $135^{\circ}$ to the prismatic/prismatic interface. Since there is a low angle grain boundary between the twin crystals with a misorientation of about $7^{\circ}$, this prismatic/prismatic interface can be described as a wall of lattice edge dislocations parallel to the line of triple junction in the $\langle a\rangle$ direction. The Burgers vector of these dislocations is $a \sqrt{ } 3$ and their separation is about $14 a$.

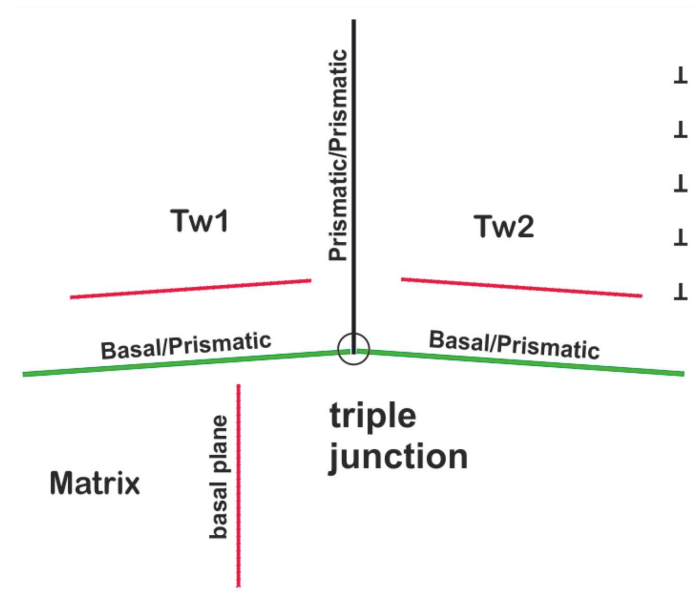

Fig. 3. Junction of three interfaces between the matrix crystal and two conjugate twins Tw1 and Tw2 in the projection along the $\langle a\rangle$ direction. The orientation of the basal planes in all tree hexagonal crystals is marked by red lines (see the online version). Dislocation wall representing the prismatic/prismatic interface is depicted in the right-hand part of the figure.

The large Burgers vector of the lattice dislocations at the interface is unstable and these dislocations can decompose into $\langle a\rangle$ dislocations or further into the Shockley partials with the Burgers vector magnitudes of $a / \sqrt{ } 3$ (see Fig. 4). Hence, the following dislocation reactions can be considered: 


$$
b=a_{3}-a_{1}
$$

and

$$
b=b_{s 2}-b_{s 1}-b_{s 3}+b_{s 2}
$$

or

$$
b=b_{s 2}+b_{s 2}+b_{s 2} .
$$

The planes of dissociation of the $\langle a\rangle$ dislocations in reaction (2) need not lie on the identical atomic plane. Total dislocation cores can be non-planar. Similarly, the three Shockley partials of reaction (3) can be also located on different atomic planes. The dislocation cores of the lattice dislocations $\boldsymbol{b}$ can thus spread over several atomic planes. The atomic structure of the dislocations $\boldsymbol{b}$ is only roughly described by the Shockley partials and need not to be the same in different metals. We wish only stress that the large Burgers vector $\boldsymbol{b}$ can decompose along the interface plane (perpendicular to $\boldsymbol{b}$ ) into several components. Only geometrically possible Burgers vectors are illustrated in Fig. 4 where the dislocation $\boldsymbol{b}$ cannot be fully characterized. Possible dissociations of the dislocation $\boldsymbol{b}$ are only the first approach to the description of the dislocation wall depicted in Fig. 3. For example, possible partial dislocations on the prismatic planes have not yet been considered.

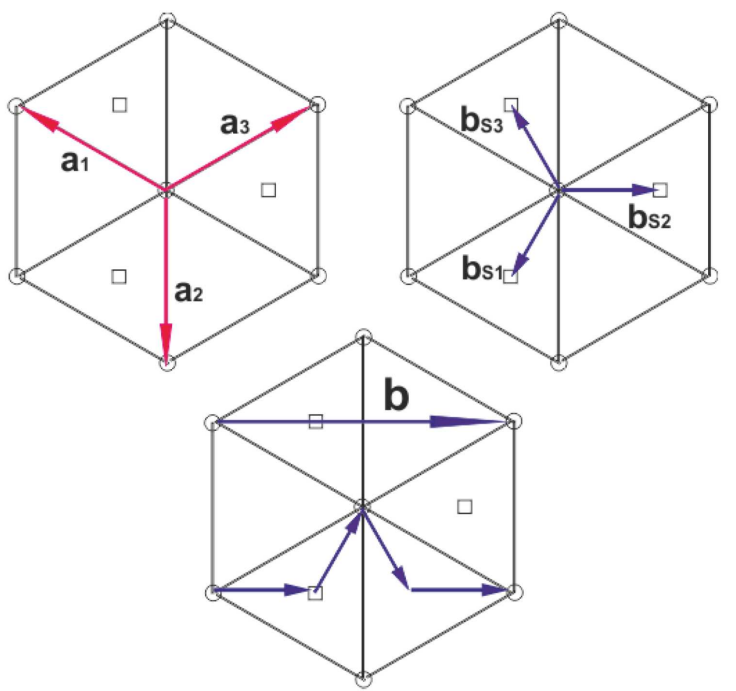

Fig. 4. Burgers vectors of the interfacial dislocations. $\boldsymbol{a}_{\mathbf{1}}, \boldsymbol{a}_{\mathbf{2}}$ and $\boldsymbol{a}_{\mathbf{3}}$ are the $\langle a\rangle$ lattice vectors then $\boldsymbol{b}_{\boldsymbol{s} \mathbf{1}}, \boldsymbol{b}_{\boldsymbol{s} \mathbf{2}}$ and $\boldsymbol{b}_{\boldsymbol{s} 3}$ are the Burgers vectors of the Shockley partials and $\boldsymbol{b}$ is the [1010] lattice vector. All these Burgers vectors lie in the basal plane. The projections of atomic positions on two alternating levels are marked by circles and squares.

\section{Discussion}

The structure of the basal/prismatic boundary described in Sect. 2 indicates that the $(10 \overline{1} 2)$ twin boundary can be easily converted to basal/prismatic boundary when the inclination of the boundary plane changes. Such a conversion does not need more than twinning disconnections and is a consequence of migration mecha- nism of the (1012) twin boundary. The conversions between these interfaces were previously demonstrated in our atomistic simulations $[10,12]$.

The encounter of two conjugate twins with a common $\langle 1 \overline{2} 10\rangle$ direction leads to interaction of basal/prismatic and prismatic/basal interfaces. Such an interaction naturally causes formation of the prismatic/prismatic boundary. The $\boldsymbol{b}_{\boldsymbol{b}}$ components with the opposite signs of both interacting interfaces can be neglected and two rows of $\boldsymbol{b}_{\boldsymbol{p}}$ will precisely compensate the misorientation between twin variants equal to about $7^{\circ}$. It is possible to speculate that the formation of the interfaces discussed here does not produce significant obstacles for twin growth and propagation. Such conclusion can be justified by relative easiness of conversions between different types of twin interfaces.

\section{Conclusions}

Special interfaces related to the $\{10 \overline{1} 2\}$ twins in hexagonal metals have been discussed. It has been demonstrated that the symmetrical twin boundaries can be converted to the basal/prismatic asymmetrical interfaces by a mechanism based on the line interfacial defects - disconnections. The same defects form also the structure of prismatic/prismatic interfaces between two conjugate twins. The dislocation structure of these planar defects has a direct impact on the properties of twins in hexagonal materials.

\section{Acknowledgments}

The authors gratefully acknowledge the financial support of the Czech Science Foundation (Project No. 1614599S).

\section{References}

[1] J.W. Christian, The Theory of Transformations in Metals and Alloys, Pergamon Press, Oxford 1965.

[2] W.B. Hutchinson, M.R. Barnett, Scr. Mater. 63, 737 (2010).

[3] A. Chapius, J.H. Driver, Acta Mater. 59, 1986 (2011).

[4] Q. Yu, J. Wang, Y. Wang, R.J. McCabe, C.N. Tome, Mater. Res. Lett. 2, 82 (2014).

[5] S. Yu, C. Liu, Y. Gao, S. Jiang, Z. Chen, Mater. Lett. 165, 185 (2016).

[6] Q. Sun, X.Y. Zhang, Y. Ren, L. Tan, J. Tu, Mater. Charact. 109, 160 (2015).

[7] A. Ostapovets, J. Bursik, K. Krahula, L. Kral, A. Serra, Philos. Mag. 97, 1088 (2017).

[8] S. Lay, G. Nouet, Philos. Mag. A 72, 603 (1995).

[9] A. Serra, D.J. Bacon, Philos. Mag. A 73, 333 (1996).

[10] A. Ostapovets, A. Serra, Acta Phys. Pol. A 128, 661 (2015).

[11] V. Paidar, A. Ostapovets, Mater. Lett. 198, 93 (2017).

[12] A. Ostapovets, A. Serra, Philos. Mag. 94, 2827 (2014). 